Mikrometer mit 4 Ocularen, eip repetirendes LampenFilar-Mikrometer mit Positionskreis und 4 Ocularen. Der Positionskreis erhält eine Theilung auf Silber, und 2 Verniers, welche die Minute angeben, und Klemme nebst Schraube zur Drehung des Mikromelers. - Um mich aber theils in den Mikrometer-Messungen an diesem Instrumente einzü̈ben, theils auch, um die Beobachtungen der Doppelsterne mit demselben gleich beginnen zu können, brackte ich das sehüne Filar-Mikrometer, das ich ron derselben kunstreichen Hand besitze, und mit welchem bisher an einem 5 füfsigen Achromate von Troughton beobachlet wurde, durch ein neues Zugrohr an den Frauentıoferschen Fefractor an, und kann also jetzt schon einige Proben von dem, was in Rücksicht auf die Messung kleiner Winkel geleistet werlen wird, darlegen.

(Der Beschlufs folgt.)

Beobachtungen des Obersten Beaufoy.

Beobachtungsplatz Bushey. Heath bei Stanmore: Breite $31^{\circ} 37^{\prime} 44^{\prime \prime} 3$, Länge $1^{\prime} 20^{\prime \prime} 93$, westlich in Zeit yon Green wich 1824 Nov. 15 . Jupiter-Tr. III. Em. $12^{\text {h }} 50^{\prime} 52^{\prime \prime} \mathrm{m} . \mathrm{Z}$.

Nov. 21. Jupiter.Tr. I. Imm. 152422
1824 Dec. 7. Jupiter-Tr. I. Imm. $13^{h} 38^{\prime} 52^{\prime \prime} \mathrm{m} . \mathrm{Z}$. Sternbedeckung.

Oct. 29. Eintritt $x$ Piscium $0^{\text {h }} 1^{\prime} 36^{\prime \prime}, 5$ Sternzeit: Ann. of $P h$. Jan. 1820 .

Beobachtungen der geraden Aufsteigungen des Mondes und benachbarter Sterne, im Jahre 1824, auf der Königsberger Sternwarte.

yon Herrn Professor und Ritter Bessel.

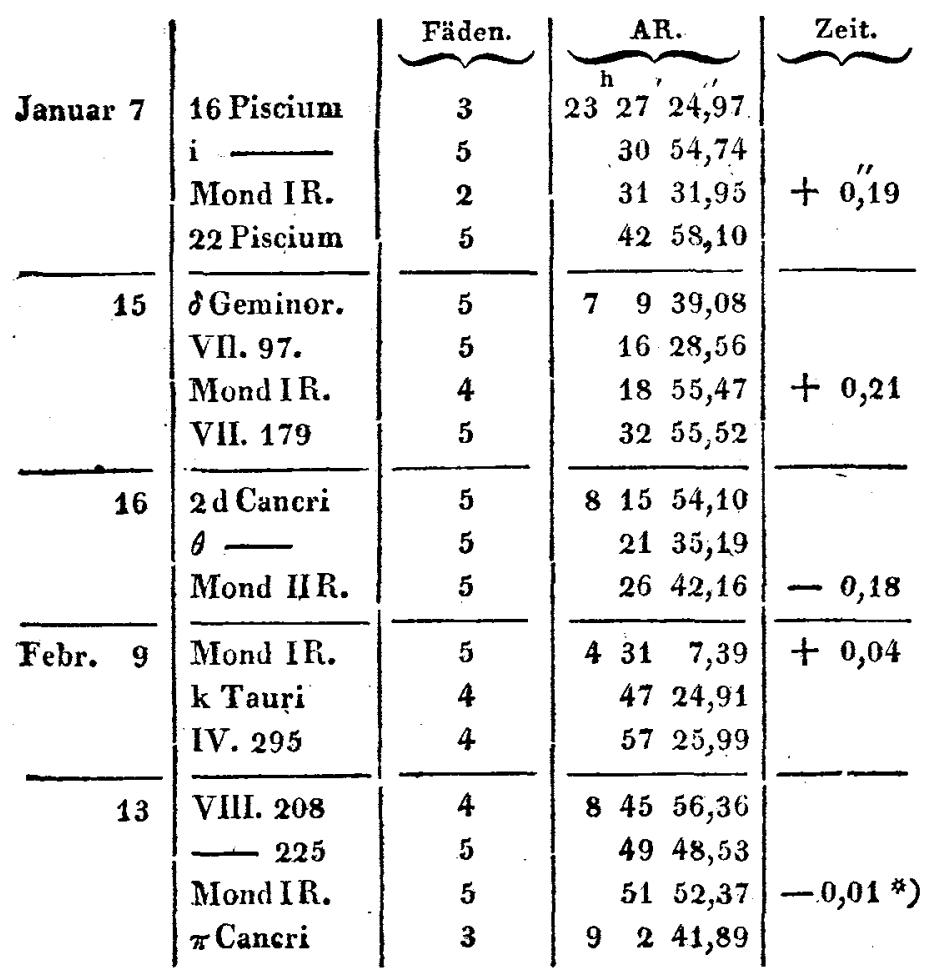

*) Diese Beobachtungen sind an sich gut, aber die Rectascensionen können etwas fehlerhaft berechnet seyn, indem der Stand der Uhr nicht an diesem Tage bestimmt werden konnte.

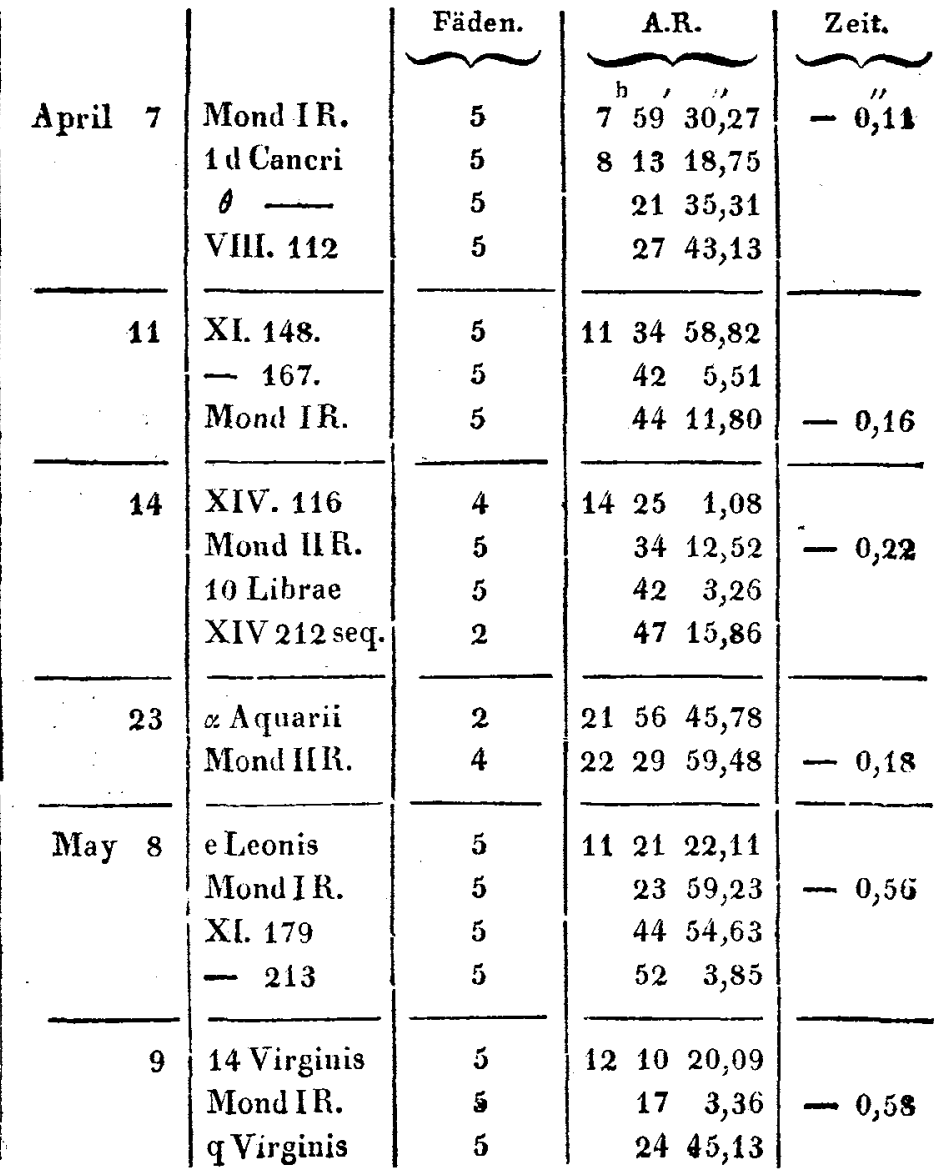




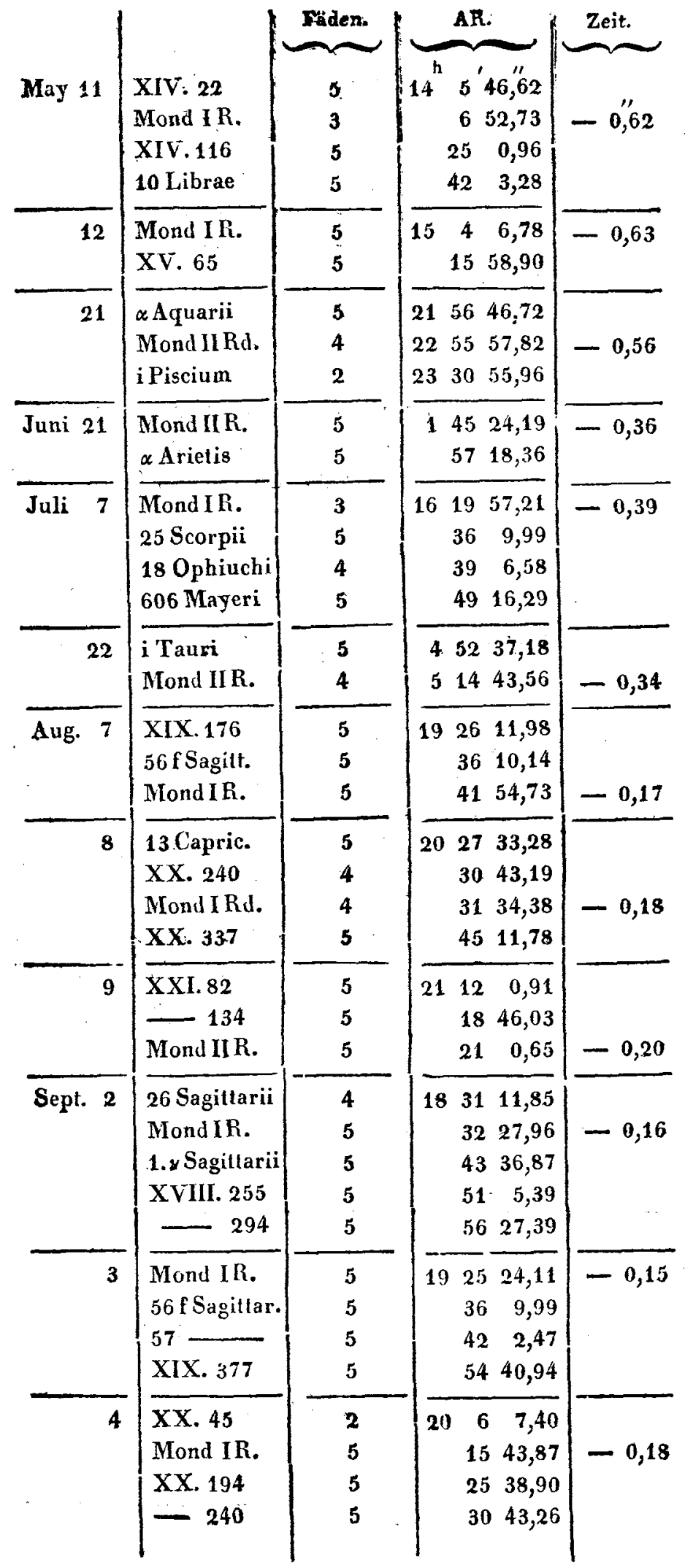

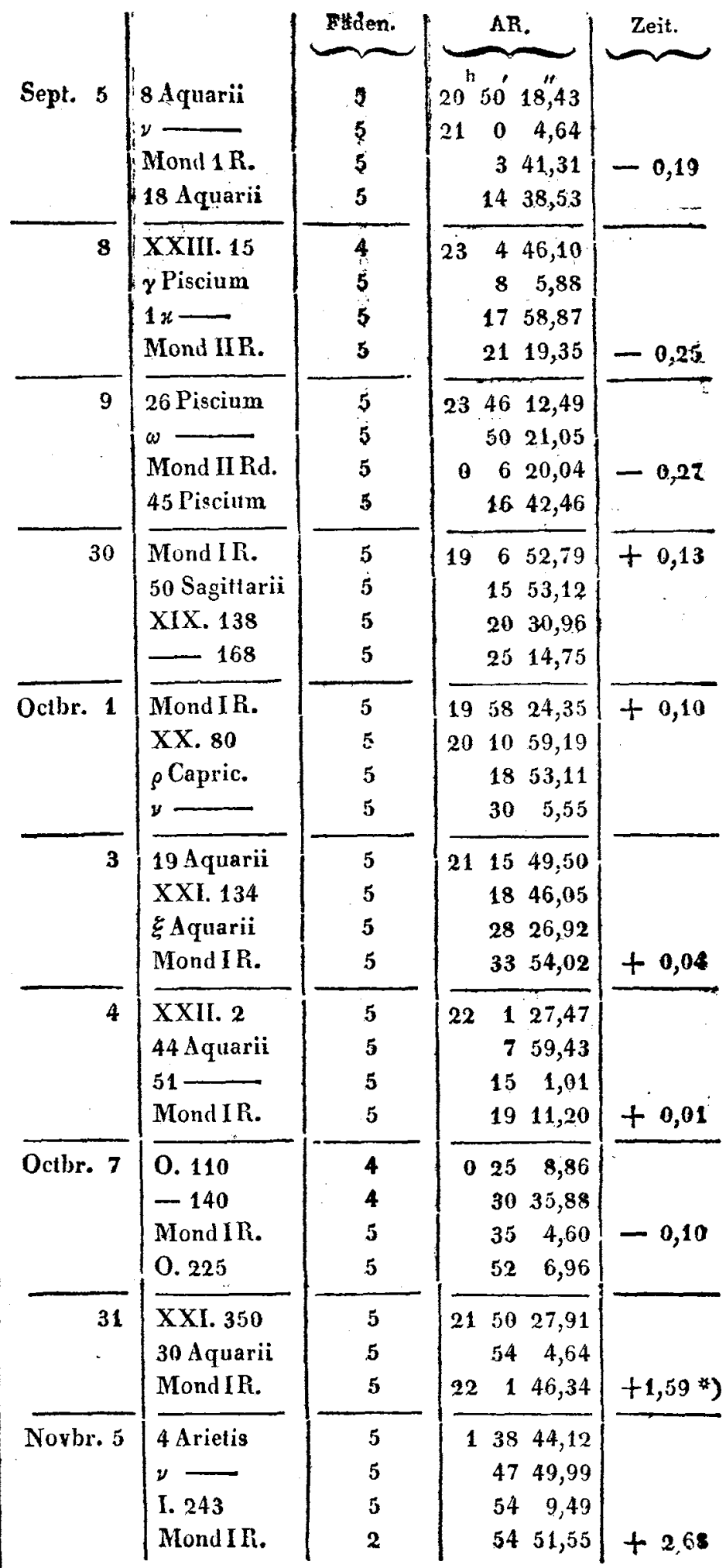

*) U̇nruhige Luft 


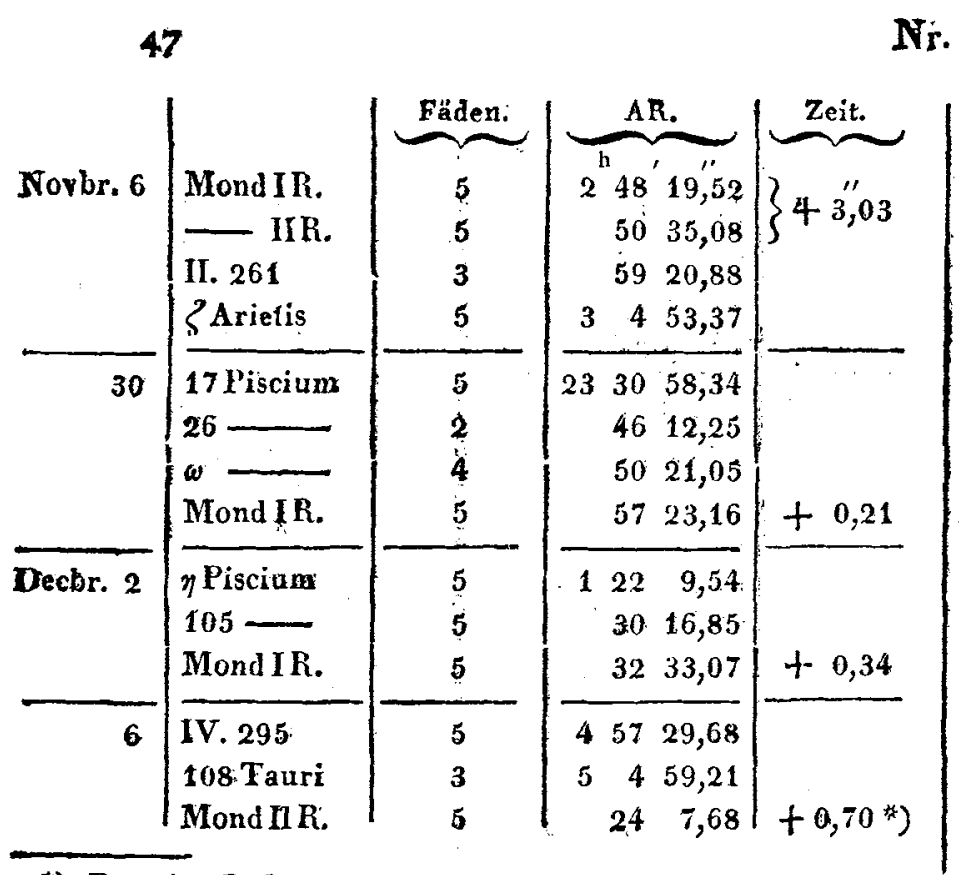

Nr. 75 .

*) Dunstige Luft.

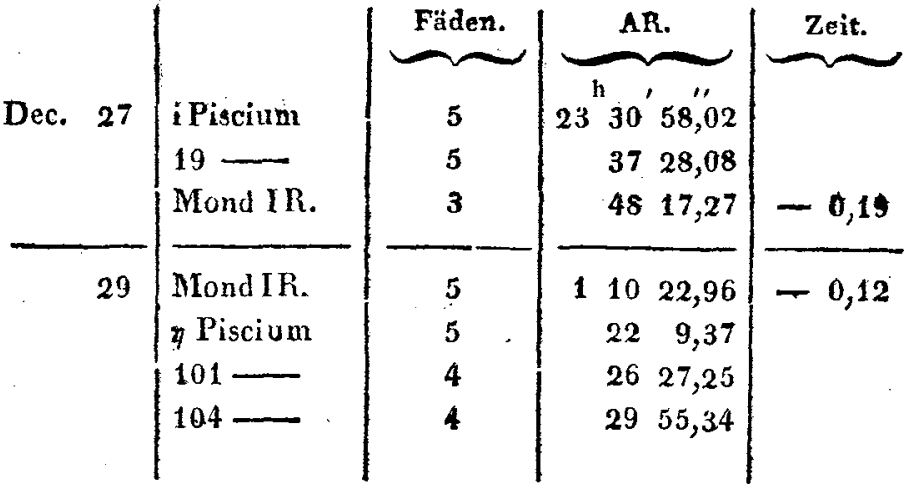

Anmerkung. Man erhält die Sternenzeit der Beobachtung des Mondes, wenn man die in der letzten Columne angegebene Zaht der geraden Aufsteigung hinzufiigt.

Bessel.

Auszug aus einem Briefe des Herrn Professor $K u l i k$ an den Herausgeber.
Grätz 1825. Jan. 27.

Ich habe die Ehre anzuzeigen, đafs ron meinem Canon logarithmorum naturalium der $12^{\text {te }}$ Bogen im Druck sey, dafs ich ferner, um dem Wunsche der p. t. Herren Subscribenten nachuukommen, den dritten Theil der Auflage auf Schreibpapier veranstaltet habe, indem ich bei den früher bereits absgedruckien Bogen das verwendete Druckpapier durch nachträgliches Leimen in Schreibpapier verwandeln liefs - ein Versuch, der über alles Erwarten sut gelungen ist. Die Beendigung des Druckes möchte daher in dem von mir angegebenen Zeitpunkte (im Mai l.J.) allerdings Statt haben. Da ich jedoch gesonnen bin, die etwanigen Druckfehler dureh eine wiederholle sorgfällige Vergleichung nach dem Druck ausfindig zu machen, und diese bei der Schreitpapierauflage durch Aufkleben der richtigen Ziffern unkenntlich machen $z u$ lassen; so dürfle dieșes die vộllige Beendigung des.Werkes bis zur MichaelisMesse 1. J. hinaussetzen, bis zu welchem Zeilpunkte ich es in den Buchhandel zu bringen hoffe.

Rälhselhaft ist es mir an der Stelle dep natïrlichen Logarithmen von $9769,9781,9787,9871,9873$ und 9907 die Briggischen Logarithmen derselben in 42 Stellen in den Schulzischen Tafeln zu treffen. - Diefs sind die alleinigen Logarithmen, um welche der Vega'sche Thesaurus vollständiger ist, der dieselben in 48 Stellen enthält. Hat sie etwa Wolfram nicht berechnet?

\section{$K \boldsymbol{l} l i k$.}

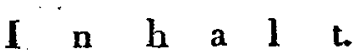

Wurm, Länge von Amsterdam und Marseille (Beschlurs.). pag. 33.

Fraunhefer, Anhang zu dem Aufsatze tibex den grofsen Refractor. pag. 35.

Struve, Nachricht von der Ankunft und Aufstellung des Refractors von Fraunhofer auf der Sternwarte aer Kaiserl. Universitä: zu Dorpat peg. 37 .

Beobachtungen des Obersten Beaufoy in Bushey-Heath. pag. 43.

Bessel, Beobachtungen dex geraden Aufsteigungen des Mondes und benachbarter Sterne, im Jahre 1824, auf der Königsberger Sternwarte. pag. 43.

Luszug aus einem Briefe des Herrn Professor Kulik an den Ferausgeber pag. 47. 